\title{
REDUCTION OF VARIABLES FOR MINIMAL SUBMANIFOLDS
}

\author{
RICHARD S. PALAIS AND CHUU-LIAN TERNG
}

\begin{abstract}
If $G$ is a compact Lie group and $M$ a Riemannian $G$ manifold, then the orbit map $\Pi: M \rightarrow M / G$ is a stratified Riemannian submersion and the well-known "cohomogeneity method" pioneered by Hsiang and Lawson $[\mathbf{H L}]$ reduces the problem of finding codimension $k$ minimal submanifolds of $M$ to a related problem in $M / G$. We show that this reduction of variables technique depends only on a certain natural Riemannian geometric property of the map $\Pi$ which we call $h$-projectability and which is shared by certain other naturally occurring and important classes of Riemannian submersions.
\end{abstract}

Suppose $\mathfrak{M}$ is a manifold of smooth maps of a compact $m$-dimensional manifold $M$ into an $n$-dimensional manifold $N$, and $J: \mathfrak{M} \rightarrow \mathbf{R}$ is a Lagrangian calculus of variations functional on $M$, i.e. $J(\varphi)=\int L(\varphi) d \mu$, where $\mu$ is a smooth measure on $M$ and $L$ is a differential operator associating a real-valued function $L(\varphi)$ to each $\varphi$ in $\mathfrak{M}$. If $x_{1}, \ldots, x_{m}$ are local coordinates in $M$ and $y_{1}, \ldots, y_{n}$ are local coordinates in $N$, then the Euler-Lagrange equations corresponding to $\delta J=0$ are a formally determined system of $n$ partial differential equations in the $m$ independent variables $x_{1}, \ldots, x_{m}$ for the $n$ unknown functions $Y_{i}=y_{i} \circ \varphi$. Finding solutions to such a problem is usually a difficult problem, and in fact the degree of difficulty is roughly measured by $m$. For example, if $m=0$ (i.e. $M$ is a point) we are really just looking for extremals of a real-valued function on $N$, and if $m=1$ we only have to solve a system of $n$ ordinary differential equations. It is therefore natural to attempt somehow to reduce the number of "effective" variables $x_{1}, \ldots, x_{m}$. One of the most successful techniques for accomplishing this goal has been to exploit some symmetry of the functional $J$. Suppose $G$ is a compact Lie group acting as a group of diffeomorphisms both of $M$ and of $N$. Then $G$ acts on the space of maps $\varphi: M \rightarrow N$ by $(g \cdot \varphi)(x)=g\left(\varphi\left(g^{-1} \cdot x\right)\right)$ and we suppose that if $\varphi$ is in $\mathfrak{M}$ then so is $g \cdot \varphi$. Let us also suppose that $J$ is $G$-invariant, i.e. $J(g \cdot \varphi)=J(\varphi)$. In these circumstances (which obtain frequently in important geometric problems) it is natural to look for solutions $\varphi$ of $\delta J=0$ which do not "break the symmetry" of the problem, i.e. those $\varphi$ belonging to the set $\mathfrak{M}^{G}=\{\varphi \in M \mid g \cdot \varphi=\varphi$ for all $g \in G\}$. Looking back to the definition we see that these are just the $G$-equivariant maps $\varphi: M \rightarrow N$.

Now it is a general fact (see $[\mathbf{P}]$ ) that $\mathfrak{M}^{G}$ is a smooth submanifold of $\mathfrak{M}$ and that if $\varphi$ is in $\mathfrak{M}^{G}$ then $\varphi$ is a critical point of $J$ if and only if it is a critical point of $J \mid \mathfrak{M}^{G}$. What makes this important is that (somewhat imprecisely) $\mathfrak{M}^{G}$ can be identified with maps of the orbit space $M / G$ into $N$, so that if $M / G$ has dimension $k$

Received by the editors November 1, 1985.

1980 Mathematics Subject Classification (1985 Revision). Primary 53A10, 53C12.

Research of the first author supported in part by NSF Grant No. MCS-8102696 and MSRI.

Research of the second author supported in part by NSF Grant No. DMS-8301928. 
(i.e. the maximum dimension orbits of $M$ have codimension $k$ ) and we are interested in equivariant solutions of our extremal problem, then we have effectively reduced the number of independent variables in our Euler-Lagrange equations from $m$ to $k$. This reduction of variables technique is usually referred to as the "cohomogeneity$k$ " method or, when $k=0$ (so $M$ is a single $G$-orbit), as the "orbit method". Perhaps the most classical example of this method (for $k=1$ ) is the derivation of an easily solved ordinary differential equation for radially symmetric harmonic functions on $\mathbf{R}^{m}$ (here $M$ is the $m$-disk, $N=\mathbf{R}, L(\varphi)=|\nabla \varphi|^{2}$, and $G=S O(m)$ ). A more sophisticated application of the cohomogeneity-1 method is H. Weyl's enormous simplification of the derivation of the Schwartzchild solution of the Einstein gravitational field equations $[\mathbf{W}]$.

In $[\mathbf{H L}]$ Hsiang and Lawson developed the cohomogeneity method for the minimal submanifold problem, and Hsiang and his students (cf. [H2] for further references) have made many striking applications, particularly of the cohomogeneity-1 method. Recently Ferus and Karcher [FK] extended the Hsiang-Lawson technique in an interesting way: they showed that the orbit foliations coming from orthogonal representations, used by Hsiang, could be replaced by foliations of Euclidean space by isoparametric hypersurfaces of spheres, thereby divorcing the cohomogeneity method from the symmetry group $G$. Based on this observation and the recent generalization of isoparametric theory to arbitrary codimensions $[\mathbf{C W}, \mathbf{T}]$, we have noted that there is a still more general (and natural) setting for reducing variables in the minimal submanifold problem. The main goal of this note is to explain this method. It turns out that the essential property for reducing variables is the existence of " $h$-projectable Riemannian submersions", which we will define below.

Let $\pi: E \rightarrow B$ be a submersion of Riemannian manifolds, $V$ the vertical distribution, defined by $V(x)=\operatorname{ker}\left(d \pi_{x}\right)$, and $\mathfrak{H}$ the horizontal distribution defined by $\mathfrak{H}(x)=$ the orthogonal complement of $V(x)$ in $T E_{x}$. Then any fiber $F=\pi^{-1}(b)$ is a submanifold of $E, V(x)=T F_{x}$, and $\mathfrak{H}(x)=v(F)_{x}$. A vector field $X$ of $E$ is called horizontal if $X(x)$ for all $x$ in $E$, and $X$ is called projectable if there is a vector field $\xi$ on $B$ such that $d \pi(X)=\xi$, i.e. $d \pi(X(x))=\xi(\pi(x))$ for all $x$ in $E$. Horizontal, projectable vector fields are also called basic. Clearly, given any smooth vector field $\xi$ on $B$ there is a unique basic field $\xi^{*}$ on $E$ with $d \pi\left(\xi^{*}\right)=\xi$. We call $\xi^{*}$ the horizontal lift of $\xi$. A curve $\alpha$ on $E$ is called horizontal if $\alpha^{\prime}(t) \in \mathfrak{H}(\alpha(t))$. Let $\alpha$ be a smooth curve on $B$ with $\alpha(0)=b$. Then given $x \in \pi^{-1}(b)$ there is a unique horizontal curve $\alpha^{*}$ on $E$ such that $\alpha^{*}(0)=x$ and $\pi\left(\alpha^{*}\right)=\alpha$. This $\alpha^{*}$ is called the horizontal lift of $\alpha$ through $x$.

A submanifold $N$ of $E$ will be called projectable if $N=\pi^{-1}(M)$ for some submanifold $M$ of $B$. A deformation $F_{t}$ of $N$ is called projectable if each $F_{t}(N)$ is projectable, and $F_{t}$ is called horizontal if each curve $F_{t}(x)$ is horizontal, or equivalently if the deformation vector field of $F_{t}$ is horizontal. $F_{t}$ is a $\pi$-invariant deformations of $N$ if it is both projectable and horizontal. Clearly if $f_{t}: M \rightarrow B$ is a deformation of $M$ then there is a unique $\pi$-invariant lifting $F_{t}: N \rightarrow E$ of $f_{t}$; namely, for each $x$ in $N, F_{t}(x)$ is the horizontal lift of the curve $f_{t}(\pi(x))$ through $x$, and the deformation field of $F_{t}$ is the horizontal lift of the deformation field of $f_{t}$. Thus there is a bijective correspondence between the $\pi$-invariant deformations of $N=\pi^{-1}(M)$ in $E$ and deformation of $M$ in $B$.

Let $\pi: E \rightarrow B$ be a Riemannian submersion [O], i.e. we assume $d \pi_{x}$ maps $\mathfrak{H}(x)$ 
isometrically onto $T B_{\pi(x)}$ for all $x$ in $E$. We define a horizontal vector field $h$ on $E$, called the fiber mean curvature vector field, as follows: if $x \in F=\pi^{-1}(b)$ then $h(x)$ is the mean curvature vector of $F$ in $E$ at $x$. The following proposition follows by a straightforward calculation.

1. Proposition. Let $\pi: E \rightarrow B$ be a Riemannian submersion, $M$ a submanifold of $B$, and $N=\pi^{-1}(M)$. Let $H$ denote the mean curvature of $M$ in $B, \hat{H}$ the mean curvature of $N$ in $E, H^{*}$ the horizontal lifting of $H$ to $N$, and $h$ the fiber mean curvature vector field in $E$. Then $\hat{H}=P(h)+H^{*}$, where $P_{x}$ is the orthogonal projection of $T E_{x}$ onto $v(N)_{x}$.

We note that if $F=\pi^{-1}(b)$ is any fiber of $E$ then we have a canonical global parallelism, the $\pi$-parallelism, in its normal bundle $v(F)=\mathfrak{H} \mid F$; namely, a section $\xi$ of $v(F)$ is $\pi$-parallel if it is projectable, i.e. if $d \pi(\xi(x))$ is independent of $x$ in $F$. Clearly, each $e \in T B_{b}$ defines a unique $\pi$-parallel field $e^{*}$, and in particular an orthonormal frame $e_{i}$ for $T B_{b}$ gives an orthonormal framing of $v(F)$ by $\pi$-parallel fields $e_{i}^{*}$. Recall that by definition the fiber mean curvature field $h$ is given along $F$ by $h(x)=\sum \operatorname{tr}\left(A_{i}(x)\right) e_{i}^{*}(x)$, where $A_{i}(x)$ is the shape operator for $F$ at $x$ in the direction $e_{i}^{*}(x)$.

2. Definition. A Riemannian submersion $\pi: E \rightarrow B$ is called $h$-projectable if the fiber mean curvature vector field $h$ is projectable. We call $\pi: E \rightarrow B$ quasihomogeneous if the eigenvalues of the shape operator of any fiber $F=\pi^{-1}(b)$ with respect to any $\pi$-parallel field $\xi$ are constant (depending only on $d \pi(\xi)$, not on $x$ in $F$ ).

It is immediate from the above formula defining $h$ that a quasi-homogeneous Riemannian submersion is $h$-projectable. We now state our main theorem.

3. THEOREM. Let $\pi: E \rightarrow B$ be an h-projectable Riemannian submersion, and $M$ a submanifold of $B$. Then a submanifold $N=\pi^{-1}(M)$ of $E$ is minimal in $E$ if and only if $N$ is a stationary point of the area functional $A$ with respect to all the $\pi$-invariant deformations of $N$ in $E$.

Proof. We have $\hat{H}=P(h)+H^{*}$ by Proposition 1. Since $h$ is projectable and $d \pi_{x}\left(v(N)_{x}\right)=v(M)_{\pi(x)}, P(h)$ and therefore $\hat{H}$ is projectable. Let $\xi$ denote the normal field $d \pi(\hat{H})$ of $M$ in $B$. Then $f_{t}(x)=\exp _{x}(t \xi(x))$ defines a deformation of $M$ in $B$ with $\xi$ as deformation field. Let $f_{t}^{*}$ be the induced $\pi$-invariant deformation of $N$ in $E$ as in Proposition 1. Then the deformation field of $f_{t}^{*}$ is $\hat{H}$. Let $A(t)=$ the area of $f_{t}^{*}(N)$, then

$$
A^{\prime}(0)=\int_{M}|\hat{H}|^{2} d v
$$

If $N$ is a critical point of $A$ with respect to all $\pi$-invariant deformations, then $A^{\prime}(0)=0$, hence $\hat{H}=0$.

Let $\pi: E^{n+k} \rightarrow B^{k}$ be an $h$-projectable Riemannian submersion. Then the above theorem implies that the minimal equation for finding $(n+r)$-dimensional $\pi$-invariant minimal submanifolds in $E$ is reduced to an equation in $r$ independent variables. To be more specific, if the fiber of $\pi$ is compact we define $v: B \rightarrow R$ by $v(b)=$ the volume of $\pi^{-1}(b)$. Then the volume of $\pi^{-1}(M)$ is the integral of the positive function $v$ with respect to the induced metric on $M$. Hence we have 
4. TheOREM. Suppose $\pi:\left(E, g_{E}\right) \rightarrow(B, g)$ is an h-projectable Riemannian submersion. Then $\pi^{-1}\left(M^{r}\right)$ is minimal in $E$ if and only if $M^{r}$ is minimal in $\left(B, g_{r}\right)$, where $g_{r}=\left(v^{2 / r}\right) g$ and $v(b)=$ the volume of $\pi^{-1}(b)$.

5. REMARK. If $\pi$ is $h$-projectable, then the vector equation $\hat{H}=P(h)+H^{*}$ in Proposition 1 is equivalent to the equation $d \pi(\hat{H})=d \pi(P(h))+H$. Hence one can reduce the problem of finding $\pi$-invariant minimal submanifolds $N=\pi^{-1}(M)$ of $E$ to the problem of finding a submanifold $M$ of $B$ with the prescribed mean curvataure vector $H=-d \pi(P(h))$. We can also reduce the problem of finding constant mean curvature hypersurfaces $N$ in $E$ to the problem of finding a hypersurface $M$ of $B$ with the prescribed mean curvature $H=-|d \pi(P(h))|+c$, for some constant $c$.

For many applications we need to consider stratified submersions.

6. Definition. Suppose $E$ is a complete Riemannian manifold, and $B=$ $\bigcup_{\alpha} B_{\alpha}$ is a stratified set such that each $B_{\alpha}$ is a Riemannian manifold. A continuous map $\pi: E \rightarrow B$ is called a stratified submersion if $E_{\alpha}=\pi^{-1}\left(B_{\alpha}\right)$ is a stratification of $E$, and $\pi_{\alpha}=\pi \mid E_{\alpha}: E_{\alpha} \rightarrow B_{\alpha}$ is a submersion for each $\alpha$. Then $\pi$ is called a stratified Riemannian submersion if each $\pi_{\alpha}$ is a Riemannian submersion, and $\pi$ is called $h$-projectable (resp. quasi-homogeneous) if the mean curvature vector of $\pi_{\alpha}^{-1}(b)$ in $E_{\alpha}$ is the mean curvature vector of $\pi_{\alpha}^{-1}(b)$ in $E$ for all $\alpha$ and $b$ in $B_{\alpha}$, and each $\pi_{\alpha}$ is $h$-projectable (resp. quasi-homogeneous).

7. Definition. $M$ is a stratified subset of a stratified set $B$ if $M \cap B_{\alpha}$ is a submanifold of $B_{\alpha}$, for each stratum $B_{\alpha}$. A deformation $f_{t}: M \rightarrow B$ is strata preserving if $f_{t}\left(M \cap B_{\alpha}\right)$ is contained in $B_{\alpha}$ for each $\alpha$. A submanifold $N$ of $E$ is $\pi$-invariant if $N$ is of the form $\pi^{-1}(M)$ for some stratified subset $M$ of $B$. Given a strata preserving deformation $f_{t}$ of $M$ into $B$, then there is a unique horizontal strata preserving lifting $F_{t}$ of $f_{t}$. We call such a deformation of $N$ a $\pi$-invariant strata preserving deformation. Then the following is a straightforward generalization of Theorem 4 above.

8. THEOREM. Let $\pi: E \rightarrow B$ be a stratified h-projectable Riemannian submersion. Then a $\pi$-invariant submanifold $N$ of $E$ is minimal in $E$ if and only if $N$ is a critical point of the area functional with respect to all the $\pi$-invariant strata preserving deformations of $N$ in $E$.

9. EXAMPLES. We will give two classes of quasi-homogeneous stratified Riemannian submersions.

(i) Let $G$ be a compact Lie group acting isometrically on a complete Riemannian manifold $E$. The mean curvature vector field $H$ of an orbit $G x$ in $E$ is clearly a $G$-equivariant normal field, and hence $H(x)$ lies in the fixed point set of the isotropy representation at $x$. But this fixed point set is the tangent space of the union of the orbits of type $\left(G_{x}\right)$. Then the orbit space $E / G$ is naturally stratified by the orbit types, and each stratum has a natural metric such that the projection map $\pi: E \rightarrow E / G$ is a quasi-homogeneous Riemannian submersion. Theorems 3,4 , and 8 for this case were proved in $[\mathbf{H L}]$.

(ii) A compact submanifold $M^{n} \subset \mathbf{R}^{n+k}$ is called isoparametric [T] if $v(M)$ is flat and the principal curvatures of $M$ with respect to any local parallel normal field are constant. Then there is a Weyl group $W$ associated to $M$ acting on $\mathbf{R}^{k}=v(M)_{x_{0}}$, and multiplicities $m_{i}$. Let $B$ be the flat simplicial cone defined by the closure of a 
Weyl chamber of $W$ in $\mathbf{R}^{k}$ with the natural simplicial stratification. It turns out that $v(M)$ has no holonomy, so given $v$ in $B$ there is a unique parallel normal field $v^{*}$ on $M$. Moreover, the parallel set $M_{v}=\left\{x+v^{*}(x) \mid x \in M\right\}$ is always a submanifold of $S^{n+k-1}$ for $v$ in $B \cap S^{k+-}$. The parallel foliation $\left\{M_{v} \mid v\right.$ in $\left.B \cap S^{k-1}\right\}$ is a singular foliation of $S^{n+k-1}$. Moreover, $B \cap S^{k-1}=S^{k-1} / W$ and $\pi: S^{n+k-1} \rightarrow S^{k-1} / W$ is a quasi-homogeneous Riemannian submersion, where $\pi(x)=v$ for $x \in M_{v}$. For $k=3$, the minimal equation of $\pi$-invariant $N^{n}$ in $S^{n+1}$ depends only on $S^{2} / W$ and the multiplicities $m_{i}$. Hence the construction of cohomogeneity-1 minimal hyperspheres in $S^{n+1}$ given in [H2] automatically produces more minimal hyperspheres in $S^{n+1}$, which may not be of cohomogeneity one.

\section{REFERENCES}

[CW] S. Carter and A. West, Isoparametric systems and transnormality, preprint.

[FK] D. Ferus and H. Karcher, Non-rotational minimal spheres and minimizing cones, preprint.

[H1] W. Y. Hsiang, On the compact homogeneous minimal submanifolds, Proc. Nat. Acad, Sci. U.S.A. 56 (1966), 5-6.

[H2] , Minimal cones and the spherical Berstein problem. I, Ann. of Math. (2) 118 (1983), 61-73; II, Invent. Math. 74 (1983), 351-369.

[HL] W. Y. Hsiang and B. H. Lawson, Jr., Minimal submanifolds of low cohomogeneity, J. Differential Geom. 5 (1971), 1-38.

[O] B. O'Neill, The fundamental equations of a submersion, Michigan Math. J. 13 (1966), 459-469.

[P] R. S. Palais, The principle of symmetric criticality, Comm. Math. Phys. 69 (1979), 19-30.

[T] C. L. Terng, Isoparametric submanifolds and their Coxeter groups, J. Differential Geom. 21 (1985), 79-107.

[W] H. Weyl, Space, time, matter, Dover, New York, 1951.

Department of Mathematics, Brandeis University, Waltham, MassachuSETTS 02254

Department of Mathematics, NORTheastern UNiVersity, Boston, MASSACHUSETTS 02115 